\title{
Spontaneous symmetry breaking in noncommutative field theories
}

\author{
H. O. Girotti* \\ Instituto de Física, Universidade Federal do Rio Grande do Sul, Caixa Postal 15051, 91501-970 Porto Alegre, RS, Brazil
}

\author{
M. Gomes ${ }^{\dagger}$ A. Yu. Petrov, ${ }^{\ddagger}$ V. O. Rivelles, ${ }^{\S}$ A. J. da Silva \\ Instituto de Física, Universidade de São Paulo, Caixa Postal 66318, 05315-970, São Paulo SP, Brazil
}

(Received 27 November 2002; published 9 June 2003)

\begin{abstract}
The spontaneous symmetry breaking of rotational $O(N)$ symmetry in noncommutative field theory is investigated in a $2+1$ dimensional model of scalar fields coupled through a combination of quartic and sextuple self-interactions. There are five possible orderings of the fields in the sextuple interaction and two for the quartic interaction. At one loop, we prove that for some choices of these orderings there is the absence of IR-UV mixing and the appearance of massless excitations. A supersymmetric extension of the model is also studied. Supersymmetry puts additional constraints on the couplings but for any given $N$ there is a Moyal ordering of the superfields for which the requirement for the existence of Goldstone bosons is satisfied. For some ordering and when $N \rightarrow \infty$ we find evidence that the model is renormalizable to all orders in perturbation theory. We also consider a generic chiral model in $3+1$ dimensions whose superpotential is invariant under local gauge transformations. We find that for any value of $N$ there is no one loop correction to the pion mass and that, at two loops, there are no pion mass corrections for slowly varying superfields so that the Goldstone theorem holds true. We also find a new purely noncommutative coupling which gives contributions starting at order $N-2$ loops.
\end{abstract}

DOI: 10.1103/PhysRevD.67.125003

PACS number(s): 11.10.Nx, 11.30.Qc

\section{INTRODUCTION}

Noncommutative quantum field theory has been intensively studied during recent years (for a review, see [1]). At first, the idea of noncommutativity was motivated by the hope that its introduction (and the ensuing nonlocality) would allow for the construction of theories with improved ultraviolet behavior. This expectation was not satisfied as noncommutative theories exhibit ultraviolet divergences of the same sort as those found in the commutative situation. However, nowadays there are other motivations, coming mainly from string theory and quantum gravity, which maintain the keen interest in the subject. Being intrinsically nonlocal these models present many unusual aspects which deserve painstaking investigation. Their main characteristic is the appearance of an infrared (IR) singularity (also referred to as UV-IR mixing [2]) which may ruin some of the properties that a well defined quantum field theory is required to possess. For instance, perturbative renormalizability is usually lost in noncommutative theories although it is regained in the supersymmetric case (see $[3,4]$ and $[5,6]$ for studies in nongauge and gauge theories, respectively).

\footnotetext{
*Email address: hgirotti@if.ufrgs.br

${ }^{\dagger}$ Email address: mgomes@fma.if.usp.br

${ }^{\ddagger}$ Also at Department of Theoretical Physics, Tomsk State Pedagogical University, Tomsk 634041, Russia. Email address: petrov@tspu.edu.ru; petrov@fma.if.usp.br

${ }^{\S}$ Also at Center for Theoretical Physics, Massachusetts Institute of Technology, Cambridge, MA 02139-4307. Email address: rivelles@lns.mit.edu; rivelles@fma.if.usp.br

"Email address: ajsilva@fma.if.usp.br
}

Another aspect that deserves better understanding is the effect of these IR singularities on the mechanism of symmetry breakdown and the appearance of Goldstone bosons in noncommutative field theory. Previous studies unveiled some interesting features. For the four dimensional linear sigma model it has been shown that, at one loop, spontaneous breakdown may occur for the $U(N)$ theory but not for $O(N)$ if $N \neq 2$. It is also worth mentioning that the Goldstone theorem holds only if the field ordering in the quartic Moyal product is consistent with local symmetry [7]. Other properties of the $O(2)$ case have also been studied in $[8,9]$ and the results for the $U(N)$ case have been extended to two loops [10]. Attempts to go to higher loops require an IR regulator which can no longer be removed [11]. Thus, only certain noncommutative extensions preserve the main features of their commutative counterparts. One may ask whether this depends upon the dimensionality of space-time or on the nature of the interaction or on both. This paper provides further understanding of this problem by means of some specific examples.

We start by studying the spontaneous symmetry breaking of the continuous $O(N)$ symmetry in a three dimensional theory and in a supersymmetric version of it, with the aim of learning about the role played by supersymmetry.

In the nonsupersymmetric case we find that there is a class of field orderings for which no UV-IR mixing occurs at one loop. Unlike the four dimensional case this does not require gauge invariant couplings. Interestingly enough, the condition for the elimination of UV divergences, in the planar sector of the pion two point function, also secures the cancellation of the would-be IR divergence, in the nonplanar sector, and, at the same time, enforces the appearance of massless excitations. This mechanism is absent in the four 
dimensional case. We remark that it is possible to extend the nonsupersymmetric model by adding a purely noncommutative interaction, i.e., one with no commutative analogue. It gives loop contributions starting at order $N-2$ so it is not relevant for our one loop results. It also exists in superspace but gives no contribution to the supersymmetric case. Its role outside the context of spontaneous symmetry breaking deserves further investigation.

While in four dimensions supersymmetry removes a dangerous IR quadratic singularity, in our three dimensional case it just further restricts the class of allowed models. Moreover, for a specific Moyal ordering we find evidence of its renormalizability to any order in the limit $N \rightarrow \infty$.

As far as noncommutative four dimensional theories are concerned, we shall be dealing with effective supersymmetric field models which arise as the low energy limit of compactified string or M theory. Usually the tree level exchange of heavy fields leads to nonrenormalizable interactions in the effective potential of the light fields. In the supersymmetric case, the decoupling of the heavy fields leads to corrections to the effective superpotential [12] and to the Kähler potential of the light fields [13]. On the other hand, the dynamics of D3-branes may be described, for slowly varying fields, by a Born-Infeld type action in which the transverse radial excitations are a set of scalar fields [14]. In the supersymmetric case one finds a chiral superfield in a specific superpotential [15]. In view of this it is natural to analyze the noncommutative versions of such theories and to study the validity of the Goldstone theorem for them, since they provide extensions of realistic string models. We assume that the interaction can be approximated by a general superpotential [17] which allows for a local $O(N)$ gauge symmetry. It will be shown that, at one loop, there are no corrections to the pion mass so that the Goldstone theorem holds true. Then, using supersymmetry alone, we go to two loops and show that for slowing varying superfields there are, again, no pion mass corrections. Thus, supersymmetry enables us to transpose the $N=2$ barrier found for the purely bosonic quartic interaction [7].

This paper is organized as follows. In Sec. II the noncommutative version of the $O(N)$ scalar model with quartic and sextuple interactions is presented, and the spontaneous symmetry breaking is studied. Section III contains a discussion of its supersymmetric version in superspace. Finally, in Sec. IV we discuss the spontaneous symmetry breaking of a chiral superfield in a generic superpotential in four dimensions. The conclusions are left to Sec. V.

\section{THE NONCOMMUTATIVE $\phi^{6}$ MODEL}

We start our analysis by considering the possible spontaneous breakdown of the $O(N)$ symmetry in a $d$ dimensional model described by the action (subscripts from the beginning and the middle of the latin alphabet run from 1 to $N$ and from 1 to $N-1$, respectively)

$$
\begin{aligned}
S= & \int d^{d} x\left[-\frac{1}{2} \phi_{a} \square \phi_{a}+\frac{\mu^{2}}{2} \phi_{a} \phi_{a}-\frac{g}{4}\left(l_{1} \phi_{a} * \phi_{a} * \phi_{b} * \phi_{b}+l_{2} \phi_{a} * \phi_{b} * \phi_{a} * \phi_{b}\right)-\frac{\lambda}{6}\left(h_{1} \phi_{a} * \phi_{a} * \phi_{b} * \phi_{b} * \phi_{c} * \phi_{c}\right.\right. \\
& \left.\left.+h_{2} \phi_{a} * \phi_{a} * \phi_{b} * \phi_{c} * \phi_{c} * \phi_{b}+h_{3} \phi_{a} * \phi_{a} * \phi_{b} * \phi_{c} * \phi_{b} * \phi_{c}+h_{4} \phi_{a} * \phi_{b} * \phi_{c} * \phi_{a} * \phi_{b} * \phi_{c}+h_{5} \phi_{a} * \phi_{b} * \phi_{c} * \phi_{a} * \phi_{c} * \phi_{b}\right)\right] .
\end{aligned}
$$

The $*$ indicates the Moyal product, which satisfies

$$
\begin{aligned}
\int & d^{d} \times \phi_{1}(x) * \phi_{2}(x) \cdots \phi_{n}(x) \\
= & \int \frac{d^{d} k_{1}}{(2 \pi)^{d}} \cdots \frac{d^{d} k_{n}}{(2 \pi)^{d}}(2 \pi)^{d} \delta^{d}\left(k_{1}+\cdots+k_{n}\right) \\
& \times \exp \left(i \sum_{i<j} k_{i} \wedge k_{j}\right) \phi_{1}\left(k_{1}\right) \phi_{2}\left(k_{2}\right) \cdots \phi_{n}\left(k_{n}\right) .
\end{aligned}
$$

Here, $k_{i} \wedge k_{j}=k_{i \mu} k_{j \nu} \theta^{\mu \nu} / 2$, and $\theta^{\mu \nu}=-\theta^{\nu \mu}$ is the antisymmetric constant matrix characterizing the underlying noncommutativity. The $l_{1}, l_{2}, h_{1}, h_{2}, \ldots, h_{5}$ are real numbers satisfying the conditions $l_{1}+l_{2}=1$ and $h_{1}+h_{2}+\cdots+h_{5}$ $=1$, so that there are two quartic and five sextuple independent interaction couplings. For constant fields, obeying the condition $\phi_{a} \phi_{a}=a^{2}$, the minimal value of the action is achieved for

$$
a^{2}=\frac{1}{2 \lambda}\left(-g+\sqrt{g^{2}+4 \mu^{2} \lambda}\right) .
$$

Assuming that the field configuration that minimizes the action is of the form $(0,0, \ldots, a)$ we redefine the fields, $\left(\pi_{i}, \sigma+a\right)$, so that the new fields $\sigma, \pi_{i}$ have zero vacuum expectation values. In terms of these new fields the action takes the form 


$$
\begin{aligned}
S= & \int d^{d} x\left\{-\frac{1}{2} \pi_{i} \square \pi_{i}-\frac{1}{2} \sigma\left(\square-m^{2}\right) \sigma-\left(2 \lambda a^{3}+g a\right) \sigma * \pi_{i} * \pi_{i}-\left(\frac{10}{3} \lambda a^{3}+g a\right) \sigma * \sigma * \sigma-\left[\left(\frac{\lambda}{6} \alpha a^{2}+\frac{g}{4} l_{1}\right) \pi_{i} * \pi_{i} * \pi_{j} * \pi_{j}\right.\right. \\
& +\left(\frac{\lambda}{6}(3-\alpha) a^{2}+\frac{g}{4} l_{2}\right) \pi_{i} * \pi_{j} * \pi_{i} * \pi_{j}+\left(\frac{\lambda}{6} a^{2} \beta+\frac{g}{2} l_{1}\right) \sigma * \sigma * \pi_{i} * \pi_{i}+\left(\frac{\lambda}{6} a^{2}(18-\beta)+\frac{g}{2} l_{2}\right) \sigma * \pi_{i} * \sigma * \pi_{i} \\
& \left.\left.+\left(\frac{5}{2} \lambda a^{2}+\frac{g}{4}\right) \sigma * \sigma * \sigma * \sigma\right]+\cdots\right\},
\end{aligned}
$$

where $m^{2}=4 \mu^{2}-2 g a^{2}=4 \lambda a^{4}+2 g a^{2}$, the ellipsis denotes terms of fifth and sixth order in the fields, $\alpha=3 h_{1}+2\left(h_{2}\right.$ $\left.+h_{3}\right)+h_{5}$, and $\beta=18 h_{1}+14 h_{2}+12 h_{3}+6 h_{4}+8 h_{5}$. Notice that condition (3) implies that the $\pi_{i}$ fields (pions) are massless in the tree approximation, in accord with the Goldstone theorem.

From the action (4) we can obtain the momentum space Feynman rules. For the propagators we have

$$
\begin{gathered}
\left\langle\sigma\left(p_{1}\right) \sigma\left(p_{2}\right)\right\rangle=(2 \pi)^{d} \delta\left(p_{1}+p_{2}\right) \frac{i}{p_{1}^{2}-m^{2}}, \\
\left\langle\pi_{i}\left(p_{1}\right) \pi_{j}\left(p_{2}\right)\right\rangle=(2 \pi)^{d} \delta\left(p_{1}+p_{2}\right) \frac{i \delta_{i j}}{p_{1}^{2}}
\end{gathered}
$$

whereas the vertices carry trigonometric factors:

$$
\begin{aligned}
\pi_{i}\left(p_{1}\right) & \pi_{j}\left(p_{2}\right) \pi_{k}\left(p_{3}\right) \pi_{l}\left(p_{4}\right) \\
\rightarrow & -i \rho_{1}\left[\cos \left(p_{1} \wedge p_{2}\right) \cos \left(p_{3} \wedge p_{4}\right) \delta_{i j} \delta_{k l}\right. \\
& +\cos \left(p_{1} \wedge p_{3}\right) \cos \left(p_{2} \wedge p_{4}\right) \delta_{i k} \delta_{j l} \\
& \left.+\cos \left(p_{1} \wedge p_{4}\right) \cos \left(p_{2} \wedge p_{3}\right) \delta_{i l} \delta_{k j}\right] \\
& -i \rho_{2}\left[\cos \left(p_{1} \wedge p_{3}+p_{2} \wedge p_{4}\right) \delta_{i j} \delta_{k l}\right. \\
& \left.+\cos \left(p_{1} \wedge p_{2}+p_{3} \wedge p_{4}\right)\right] \delta_{i k} \delta_{j l} \\
& \left.+\cos \left(p_{1} \wedge p_{2}+p_{4} \wedge p_{3}\right) \delta_{i l} \delta_{k j}\right] \\
\pi_{j}\left(p_{1}\right) & \pi_{j}\left(p_{2}\right) \sigma\left(p_{3}\right) \sigma\left(p_{4}\right) \\
\rightarrow & -i\left[\rho_{3} \cos \left(p_{1} \wedge p_{2}\right) \cos \left(p_{3} \wedge p_{4}\right)\right. \\
& \left.+\rho_{4} \cos \left(p_{1} \wedge p_{3}+p_{2} \wedge p_{4}\right)\right], \\
\sigma\left(p_{1}\right) * \pi_{i}\left(p_{2}\right) * & \pi_{i}\left(p_{3}\right) \rightarrow-i\left(4 \lambda a^{3}+2 g a\right) \cos \left(p_{2} \wedge p_{3}\right),
\end{aligned}
$$

where $\quad \rho_{1}=(4 \lambda / 3) a^{2} \alpha+2 g l_{1}, \quad \rho_{2}=(3-\alpha)(4 \lambda / 3) a^{2}$ $+2 g l_{2}, \rho_{3}=(2 \lambda / 3) a^{2} \beta+2 g l_{1}$, and $\rho_{4}=(2 \lambda / 3) a^{2}(18-\beta)$ $+2 \mathrm{gl}_{2}$.

To study quantum corrections to the pion's mass we should introduce the renormalizations $\phi_{a} \rightarrow\left(1+\delta_{z}\right)^{1 / 2} \phi_{a}$, $\mu^{2} \rightarrow \mu^{2}+\delta_{\mu^{2}}, \lambda \rightarrow \lambda+\delta_{\lambda}$ and $g \rightarrow g+\delta_{g}$. The reparametrizations of the "relative" couplings $l$ and $h$ (i.e., $l_{i} \rightarrow l_{i}+\delta l_{i}$ and $h_{i} \rightarrow h_{i}+\delta h_{i}$ with $\left.\Sigma \delta l_{i}=\Sigma \delta h_{i}=0\right)$ are, of course, also done but they do not show up in our calculations. The corresponding counterterm Lagrangian is, therefore,

$$
\begin{aligned}
L_{c t}= & -\frac{1}{2} \pi_{i}\left(\delta_{z} \square-\delta_{\mu^{2}}+\delta_{g} a^{2}+\delta_{\lambda} a^{4}\right) \pi_{i} \\
& -\frac{1}{2} \sigma\left(\delta_{z} \square-\delta_{\mu^{2}}+3 \delta_{g} a^{2}+5 \delta_{\lambda} a^{4}\right) \sigma \\
& +\left(\delta_{\mu^{2}} a-\delta_{g} a^{3}-\delta_{\lambda} a^{5}\right) \sigma+\cdots,
\end{aligned}
$$

where the ellipsis stands for other interaction terms obtained from Eq. (4) by replacing $\lambda$ and $g$ by $\delta_{\lambda}$ and $\delta_{g}$, respectively. Some of these counterterms are depicted in Fig. 1, where the $\sigma$ and $\pi$ propagators are represented by continuous and dashed lines, respectively.

We begin our one loop analysis of spontaneous breaking of the $O(N)$ symmetry in $2+1$ dimensions by considering the condition for the vanishing of the vacuum expectation value of $\sigma$ (the gap equation). It is found to read

$$
\begin{aligned}
\delta_{\mu^{2}}-\delta_{g} a^{2}-\delta_{\lambda} a^{4}= & \left(10 a^{2} \lambda+3 g\right) \int \frac{d^{3} k}{(2 \pi)^{3}} \frac{i}{k^{2}-m^{2}} \\
& +(N-1)\left(2 a^{2} \lambda+g\right) \int \frac{d^{3} k}{(2 \pi)^{3}} \frac{i}{k^{2}},
\end{aligned}
$$

thus fixing the above linear combination of counterterms. [In the remainder of this paper all superficially divergent integrals are implicitly assumed to be regularized. The precise form of the regularization is irrevelant, as far as it obeys the usual (additive) rules employed in the calculation of Feynman amplitudes.] It should be observed that this combination coincides with the $\pi$ field mass counterterm. The gap equation (11) is graphically represented in Fig. 2.

We next examine the one loop corrections to the pion's two point function which are shown in Fig. 3. We denote the contribution from the graph with the dashed line loop as $I_{1}(p)$, that from the solid line loop as $I_{2}(p)$, and that from the loop with two internal lines as $I_{3}(p)$. One has that 


$$
\begin{aligned}
I_{1}(p)= & \delta^{i j}\left(\frac{\lambda a^{2}}{3}[(2 N-4) \alpha+12]+g\left(N l_{1}+2 l_{2}\right)\right) \int \frac{d^{3} k}{(2 \pi)^{3}} \frac{1}{k^{2}}+\delta^{i j}\left(\frac{2 \lambda a^{2}}{3}[\alpha+(3-\alpha)(N-1)]+g l_{1}+g l_{2}(N-1)\right) \\
& \times \int \frac{d^{3} k}{(2 \pi)^{3}} \frac{e^{2 i k \wedge p}}{k^{2}} \\
I_{2}(p)= & \delta^{i j}\left(\frac{1}{3} \lambda a^{2} \beta+g l_{1}\right) \int \frac{d^{3} k}{(2 \pi)^{3}} \frac{1}{k^{2}-m^{2}}+\delta^{i j}\left(\frac{1}{3} \lambda a^{2}(18-\beta)+g l_{2}\right) \int \frac{d^{3} k}{(2 \pi)^{3}} \frac{e^{2 i k \wedge p}}{k^{2}-m^{2}} \\
I_{3}(p)= & 4 \delta^{i j}\left(2 \lambda a^{3}+g a\right)^{2} \int \frac{d^{3} k}{(2 \pi)^{3}} \frac{\cos ^{2}(k \wedge p)}{(k+p)^{2}\left(k^{2}-m^{2}\right)}
\end{aligned}
$$

Therefore, at one loop altogether we have

$$
\begin{gathered}
\delta^{i j}\left\{\left(\frac{2 \lambda a^{2}}{3}[6-\alpha+(\alpha-3)(N-1)]+g\left(N l_{1}+2 l_{2}-N+1\right)\right) \int \frac{d^{3} k}{(2 \pi)^{3}} \frac{1}{k^{2}}-\left(10 \lambda a^{2}+\left(3-l_{1}\right) g-\frac{\lambda a^{2}}{3} \beta\right)\right. \\
\quad \times \int \frac{d^{3} k}{(2 \pi)^{3}} \frac{1}{k^{2}-m^{2}}+\left(\frac{2 \lambda a^{2}}{3}[\alpha+(3-\alpha)(N-1)]+g l_{1}+g l_{2}(N-1)\right) \int \frac{d^{3} k}{(2 \pi)^{3}} \frac{\cos (2 k \wedge p)}{k^{2}} \\
\left.\quad+\left(\frac{1}{3} \lambda a^{2}(18-\beta)+g l_{2}\right) \int \frac{d^{3} k}{(2 \pi)^{3}} \frac{\cos (2 k \wedge p)}{k^{2}-m^{2}}+4\left(2 \lambda a^{3}+g a\right)^{2} \int \frac{d^{3} k}{(2 \pi)^{3}} \frac{\cos ^{2}(k \wedge p)}{(k+p)^{2}\left(k^{2}-m^{2}\right)}+\delta_{z} p^{2}\right\}
\end{gathered}
$$

As $\int d^{3} k\left[1 /\left(k^{2}-m^{2}\right)-1 / k^{2}\right]$ is finite, from the above results it follows that the UV divergences in the planar contributions may be eliminated if

$$
\begin{aligned}
& \frac{2 \lambda a^{2}}{3}[6-\alpha+(\alpha-3)(N-1)]+g\left(N l_{1}+2 l_{2}-N+1\right) \\
& \quad=10 \lambda a^{2}+\left(3-l_{1}\right) g-\frac{\lambda a^{2}}{3} \beta .
\end{aligned}
$$

To prevent the IR-UV mixing in the nonplanar parts also one must have

$$
\begin{aligned}
& \frac{2 \lambda a^{2}}{3}[\alpha+(3-\alpha)(N-1)]+g l_{1}+g l_{2}(N-1) \\
& \quad=-\left(\frac{1}{3} \lambda a^{2}(18-\beta)+g l_{2}\right) .
\end{aligned}
$$

Actually, we notice that Eq. (16) implies Eq. (17) and vice versa, i.e., UV divergences and IR-UV mixing in the pion two point functions are simultaneously eliminated. Moreover, in addition to leading to a finite result, condition (16) [or (17)] also ensures that the resulting expression vanishes for $p=0$. Indeed, after imposing Eq. (16), the expression (15) may be rewritten as

$$
\begin{aligned}
\delta^{i j}\{( & \left(\frac{1}{3} \lambda a^{2}(18-\beta)+g l_{2}\right) \int \frac{d^{3} k}{(2 \pi)^{3}} \frac{m^{2} \cos (2 p \wedge k)}{k^{2}\left(k^{2}-m^{2}\right)} \\
& -\left(10 \lambda a^{2}+\left(3-l_{1}\right) g-\frac{\lambda a^{2}}{3} \beta\right) \int \frac{d^{3} k}{(2 \pi)^{3}} \frac{m^{2}}{k^{2}\left(k^{2}-m^{2}\right)} \\
& \left.+4\left(2 \lambda a^{3}+g a\right)^{2} \int \frac{d^{3} k}{(2 \pi)^{3}} \frac{\cos ^{2}(k \wedge p)}{(k+p)^{2}\left(k^{2}-m^{2}\right)}\right\}
\end{aligned}
$$

which is finite and vanishes at $p=0$. This result is a peculiarity of the $2+1$ dimensional world where there are at most linear UV divergences (for the one loop graphs that we have considered). To see why this is so, notice that in the commutative version of the model the sum

$$
\text { pion mass counterterm }+I_{1}(0)+I_{2}(0)
$$

is finite, namely, no infinite pion wave function renormalization is necessary. Now, it is clear that if by adjusting the parameters of the model a subsum of the integrals occurring

$$
\begin{array}{cc}
i \ldots-\ldots j & =-i \delta^{i j}\left(-\delta_{\mu^{2}}+\delta_{g} a^{2}+\delta_{\lambda} a^{4}-\delta_{z} p^{2}\right) \\
\times & =-i\left(\delta_{\mu^{2}} a-\delta_{g} a^{3}-\delta_{\lambda} a^{5}\right) \\
\times & =-i\left(-\delta_{\mu^{2}}+3 \delta_{g} a^{2}+5 \delta_{\lambda} a^{4}-\delta_{z} p^{2}\right)
\end{array}
$$

FIG. 1. Structure of counterterms in the noncommutative $\phi^{6}$ model. Continuous and dashed lines represent the $\sigma$ and $\pi_{i}$ fields, respectively. 


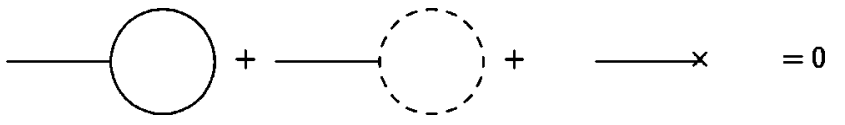

FIG. 2. Gap equation in the noncommutative $\phi^{6}$ model.

in Eq. (19) is made finite then with the same choice of parameters the subsum of the remaining integrals will also be finite. In the noncommutative model that we are dealing with the two sets of integrals correspond to the planar and nonplanar parts of the graphs contributing to Eq. (19). The appearance of the Goldstone bosons is a straightforward consequence of the fact that the pion two point function is finite at $p=0$ (no infrared divergence). Therefore $p$ can be made zero directly in the integrands of Eq. (15), leading to the same expression as in the commutative case.

In $3+1$ dimensions $\lambda=0$ is necessary for renormalizability, the divergences are quadratic, and although Eq. (15) still vanishes at $p=0$ the UV convergence of the planar part demands, for general $p$, that the left hand side of Eq. (16) be equal to

$$
\frac{2\left(2 \lambda a^{3}+g a\right)^{2}}{m^{2}}
$$

This new equation strongly restricts the dynamics so that the class of allowed models requires $N=2$ and $l_{1}=2$ as proved in [7].

Returning to the model under analysis, we observe that for a given $N$ Eq. (16) expresses $\lambda$ in terms of $g$, for general values of $\alpha, \beta$, and $l_{1}$. However, for arbitrary nonzero $\lambda$ and $g$ Eq. (16) is satisfied if

$$
l_{1}=\frac{N}{N-1} \text { and } \beta=12+6 N-2 \alpha(N-2) .
$$

To complete the investigation of the existence of the IR-UV mixing, one has to study also the behavior of the other $n$ point functions that have positive superficial degrees of divergence. In the case of the $\sigma$ field two point function one finds that the vanishing of infrared quadratic divergences now demands that

$$
10 \lambda a^{2}+g+(N-1)\left(\frac{\lambda a^{2}}{3}(18-\beta)+g l_{2}\right)=0
$$

The discussion may be extended to the one loop calculations of the three and four point functions. One finds that the relevant contributions arise from the vertices with five and six fields. In that situation, the new parameters which come into play are enough to eliminate the possible IR-UV mixing. One may conclude that, as far as renormalizability is concerned, the model is consistent up to one loop, for any value of $N$.

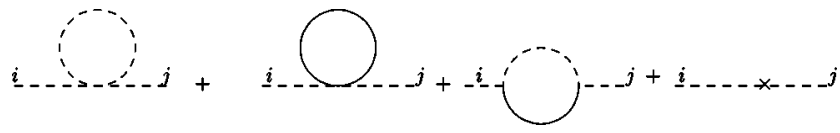

FIG. 3. One-loop contributions to the pion two-point function in the noncommutative $\phi^{6}$ model.

It is possible to generalize the action (1) by adding to it an interaction that is purely noncommutative in character and whose existence is due to the $O(N)$ symmetry. For a given $N$ it has the form

$$
\int d^{d} x \epsilon^{a_{1} \cdots a_{N}} \phi_{a_{1}} * \cdots * \phi_{a_{N}}
$$

Clearly, Eq. (23) vanishes in the commutative case, while in the noncommutative one it is only nonvanishing for odd $N$. After the shift, it contributes to Eq. (4) with a term like

$$
N \epsilon^{i_{1} \cdots i_{N-1}} \pi_{i_{1}} * \cdots * \pi_{i_{N-1}} * \sigma
$$

For $N=4$ this contribution starts at two loops, whereas for general $N$ it starts at $N-2$ loops. So the modification of the action implied by the addition of Eq. (23) does not alter our former conclusions, which were derived at one loop level, but may become relevant at higher loop levels.

\section{SUPERSYMMETRIC VERSION IN 2+1 DIMENSIONS}

A simple supersymmetric extension of the model studied in the last section is provided by the superfield action

$$
\begin{aligned}
S= & \int d^{5} z\left(\frac{1}{2} \Phi_{a} D^{2} \Phi_{a}+\frac{1}{2} \mu \Phi_{a} \Phi_{a}\right. \\
& \left.-\frac{g}{4}\left[f \Phi_{a} * \Phi_{a} * \Phi_{b} * \Phi_{b}+(1-f) \Phi_{a} * \Phi_{b} * \Phi_{a} * \Phi_{b}\right]\right) .
\end{aligned}
$$

Here, $D=\partial / \partial \theta-i \bar{\theta} \phi, \bar{D}=\gamma_{0} D, \theta_{\alpha}, \alpha=1,2\left(\bar{\theta} \equiv \theta \gamma^{0}\right)$, are Grassmann variables, $D^{2}=\frac{1}{2} \bar{D} D$, and the superfield $\Phi$ has the decomposition

$$
\Phi=\phi+\bar{\theta} \psi+\frac{\bar{\theta} \theta}{2} F
$$

where $\psi$ is an $N$ component Majorana spinor and $\phi$ and $F$ are ( $N$ component) scalar fields. In terms of field components the Lagrangian reads 


$$
\begin{aligned}
\mathcal{L}= & \frac{1}{2} \partial_{\mu} \phi \partial^{\mu} \phi+\frac{i}{2} \bar{\psi} \theta \psi+\frac{1}{2} F^{2}-\mu F \phi+\frac{\mu}{2} \bar{\psi} \psi+\frac{g}{2} f\left[F_{a} * \phi_{a}+\phi_{a} * F_{a}\right] * \phi_{b} * \phi_{b}+\frac{g}{2}(1-f)\left[F_{a} * \phi_{b}+\phi_{a} * F_{b}\right] * \phi_{a} * \phi_{b} \\
& -\frac{g}{2} f \bar{\psi}_{a} * \psi_{a} * \phi_{b} * \phi_{b}-\frac{g}{4} f\left[\bar{\psi}_{a} * \phi_{a}+\phi_{a} * \bar{\psi}_{a}\right] *\left[\psi_{b} * \phi_{b}+\phi_{b} * \psi_{b}\right]-\frac{g}{2}(1-f)\left[\bar{\psi}_{a} * \psi_{b} * \phi_{a} * \phi_{b}-\psi_{a} * \bar{\psi}_{b} * \phi_{a} * \phi_{b}\right. \\
& \left.+\bar{\psi}_{a} * \phi_{b} * \psi_{a} * \phi_{b}\right] .
\end{aligned}
$$

By integrating out $F$ (or, alternatively, by eliminating it through the use of the equations of motion) one obtains quartic and sextuple self-interactions with definite strengths.

In what follows we will work directly with the action (25). Classically, it possesses $O(N)$ symmetry and its potential part has a minimum at a constant value of the superfield $|a|=\sqrt{\mu / g}$. As in the nonsupersymmetric case, to break the $O(N)$ symmetry we suppose that one of fields (for the sake of concreteness, $\Phi_{N}$ ) has a nonzero vacuum expectation value $a$. All other fields have zero vacuum expectation values. Then we make the changes $\Phi_{a}=\pi_{i}$ for $i \neq N$ and $\Phi_{N}=\sigma+a$. The superfields $\pi_{i}, \sigma$ have zero vacuum expectation values. In terms of the new variables the action can be cast as

$$
\begin{aligned}
S\left[\pi_{i}, \sigma\right]= & \int d^{5} z\left(\frac{1}{2} \pi_{i} D^{2} \pi_{i}+\frac{1}{2} \sigma\left(D^{2}-2 \mu\right) \sigma-\frac{g}{4} \sigma * \sigma * \sigma * \sigma-g a \sigma * \sigma * \sigma-g a \pi_{i} * \pi_{i} * \sigma\right. \\
& \left.-\frac{g}{4}\left[f \pi_{i} * \pi_{i} * \pi_{j} * \pi_{j}+(1-f) \pi_{i} * \pi_{j} * \pi_{i} * \pi_{j}\right]-\frac{g}{2}\left[f \pi_{i} * \pi_{i} * \sigma * \sigma+(1-f) \pi_{i} * \sigma * \pi_{i} * \sigma\right]\right) .
\end{aligned}
$$

The counterterm Lagrangian is

$$
\begin{aligned}
L_{c t}= & \frac{1}{2} \delta_{z} \pi_{i} D^{2} \pi_{i}+\frac{1}{2} \delta_{z} \sigma D^{2} \sigma+\frac{1}{2}\left(-a^{2} \delta_{g}+\delta_{\mu}\right) \pi_{i} \pi_{i}+\frac{1}{2}\left(-3 a^{2} \delta_{g}+\delta_{\mu}\right) \sigma^{2}+\left(\delta_{\mu} a-\delta_{g} a^{3}\right) \sigma-\frac{\delta_{g}}{4} \sigma * \sigma * \sigma * \sigma-\delta_{g} \sigma * \sigma * \sigma a \\
& -\delta_{g} \pi_{i} * \pi_{i} * \sigma a-\frac{\delta_{g}}{4}\left[f \pi_{i} * \pi_{i} * \pi_{j} * \pi_{j}+(1-f) \pi_{i} * \pi_{j} * \pi_{i} * \pi_{j}\right]-\frac{\delta_{g}}{2}\left[f \pi_{i} * \pi_{i} * \sigma * \sigma+(1-f) \pi_{i} * \sigma * \pi_{i} * \sigma\right], \quad(29)
\end{aligned}
$$

the renormalization being done through the replacements $\pi_{i} \rightarrow\left(1+\delta_{z}\right) \pi_{i}, \quad \sigma \rightarrow\left(1+\delta_{z}\right) \sigma, \quad \mu \rightarrow \mu+\delta \mu, \quad g \rightarrow g+\delta g$, and $f \rightarrow f+\delta f$. The propagators corresponding to the action (28) at $a=\sqrt{\mu / g}$ are

$$
\begin{gathered}
\left\langle\pi_{i}\left(x_{1}, \theta_{1}\right) \pi_{j}\left(x_{2}, \theta_{2}\right)\right\rangle=-i \frac{D^{2}}{\square} \delta_{i j} \delta^{5}\left(z_{1}-z_{2}\right), \\
\left\langle\sigma\left(x_{1}, \theta_{1}\right) \sigma\left(x_{2}, \theta_{2}\right)\right\rangle=-i \frac{D^{2}+m}{\square+m^{2}} \delta_{i j} \delta^{5}\left(z_{1}-z_{2}\right),
\end{gathered}
$$

where $\delta^{5}\left(z_{1}-z_{2}\right)=\delta^{3}\left(x_{1}-x_{2}\right) \delta\left(\bar{\theta}_{1}-\bar{\theta}_{2}\right) \delta\left(\theta_{1}-\theta_{2}\right)$ and $m$ $=2 g a^{2}$. We adopt a graphical notation similar to the one in Sec. II. Thus, we represent the $\pi$ field propagator by a dashed line, and the $\langle\sigma \sigma\rangle$ propagator by a solid one. The trigonometric factors associated with the cubic and quartic vertices are the same as in the previous section, and we will not list them here. Now, however, we have to make the following identifications:

$\pi$ field bilinear counterterm: $i \delta_{i j}\left(\delta_{\mu}-\delta_{g} a^{2}+\delta_{z} D^{2}\right)$,

$\sigma$ field bilinear counterterm: $i\left(\delta_{\mu}-3 \delta_{g} a^{2}+\delta_{z} D^{2}\right)$,

$\sigma$ field tadpole counterterm: $i\left(-\delta_{g} a^{3}+\delta_{\mu} a\right)$.

The vanishing of the vacuum expectation value of the $\sigma$ superfield leads to the the gap equation

$$
\begin{aligned}
3 g a \int & \frac{d^{3} k}{(2 \pi)^{3}} \frac{1}{k^{2}-m^{2}}+g a(N-1) \\
& \times \int \frac{d^{3} k}{(2 \pi)^{3}} \frac{1}{k^{2}}+i\left(\delta_{\mu} a-\delta_{g} a^{3}\right)=0 .
\end{aligned}
$$

As before, this condition is not affected by the noncommutativity and implies that

$$
\begin{aligned}
\delta_{\mu} a-\delta_{g} a^{3}= & g a(N-1) \int \frac{d^{3} k}{(2 \pi)^{3}} \frac{i}{k^{2}} \\
& +3 g a \int \frac{d^{3} k}{(2 \pi)^{3}} \frac{i}{k^{2}-m^{2}} .
\end{aligned}
$$

Concerning the $\pi$ superfield two point function, one finds that, graphically, the condition for the cancellation of divergent corrections to the $\left\langle\pi_{i} \pi_{j}\right\rangle$ propagator is the same as in Fig. 3. Adopting the same convention as in the previous case, it reads

$$
I_{1}(p)+I_{2}(p)+I_{3}(p)+I_{c t}=\text { finite } .
$$

An explicit calculation yields 


$$
\begin{aligned}
& I_{1}(p)=\delta^{i j} g[f N+2(1-f)] \int \frac{d^{3} k}{(2 \pi)^{3}} \frac{1}{k^{2}} \\
& +\delta^{i j} g[f+(1-f)(N-1)] \int \frac{d^{3} k}{(2 \pi)^{3}} \frac{e^{2 i k \wedge p}}{k^{2}}, \\
& I_{2}(p)=\delta^{i j} g f \int \frac{d^{3} k}{(2 \pi)^{3}} \frac{1}{k^{2}-m^{2}} \\
& +\delta^{i j} g(1-f) \int \frac{d^{3} k}{(2 \pi)^{3}} \frac{e^{2 i k \wedge p}}{k^{2}-m^{2}}, \\
& I_{3}(p)=2 g^{2} a^{2} \delta^{i j} \int \frac{d^{3} k}{(2 \pi)^{3}} \frac{\cos ^{2}(k \wedge p)}{k^{2}\left(k^{2}-m^{2}\right)}\left(D^{2}+m\right) .
\end{aligned}
$$

Hence, the coefficient of $D^{2}$ turns out to be

$$
\delta_{z}=2 g^{2} a^{2} \int \frac{d^{3} k}{(2 \pi)^{3}} \frac{\cos ^{2}(k \wedge p)}{k^{2}\left(k^{2}-m^{2}\right)}=-\frac{g^{2} a^{2}}{\sqrt{2} \pi m}+O(\theta p) .
$$

This integral is finite and nonsingular at $p \rightarrow 0$, and, therefore, we have only a finite wave function renormalization for the $\pi_{i}$ fields.

The correction to the mass of the pion superfield, $S_{m}$, is the sum of the $D^{2}$-independent parts of $I_{1}(p), I_{2}(p), I_{3}(p), I_{c t}$. It is given by the relation

$$
\begin{aligned}
S_{m}= & \int \frac{d^{3} k}{(2 \pi)^{3}}\left\{\frac{1}{k^{2}}(2-N)(1-f)+\frac{1}{k^{2}-m^{2}}(f-2)\right. \\
& \left.+\frac{e^{2 i k \wedge p}}{k^{2}}(N-2)(1-f)+\frac{e^{2 i k \wedge p}}{k^{2}-m^{2}}(2-f)\right\}
\end{aligned}
$$

and is both UV finite and without dangerous IR-UV mixing if $(2-N)(1-f)=2-f$, i.e., if $f=N /(N-1)$. At this value of $f$, we get

$$
S_{m}=m^{2} \frac{2-N}{N-1} \int \frac{d^{3} k}{(2 \pi)^{3}} \frac{1-\cos (2 k \wedge p)}{k^{2}\left(k^{2}-m^{2}\right)},
$$

which vanishes at $p=0$, as required.

The above result suggests that, since $f \rightarrow 1$ for large $N$, only the first quartic ordering in Eq. (25) survives in this limit. This indicates that the use of the $1 / N$ expansion becomes appropriate and that by proceeding along the lines described in [4] one may prove renormalizability to all orders.

Our analysis can easily be extended to the case in which the basic superfields belong to a representation of the $U(N)$ group. For the fundamental representation, similarly to the nonsupersymmetric situation, one finds that Goldstone's theorem holds if a gauge invariant Moyal ordering of the basic fields is adopted. For the adjoint representation there are additional difficulties due to the matrix character of the superfields.

\section{GENERIC CHIRAL MODEL IN 3+1 DIMENSIONS}

As discussed in Sec. I, we next consider a generic $O(N)$ chiral model whose action is given by

$$
S=\int d^{8} z \bar{\Phi}_{a} \Phi_{a}-\left(\int d^{6} z W\left(\Phi_{a}\right)+\text { H.c. }\right),
$$

where $W$ is the superpotential. In order to be invariant under local transformations we must have an even number of superfields, so that we can take complex combinations of them. As for the Moyal ordering it must be of the form $\cdots * \Phi_{a} * \Phi_{a} * \Phi_{a+1} * \Phi_{a+1} * \ldots$. We then restrict $N$ to be even and the superpotential to be given by

$$
W\left(\Phi_{a}\right)=\left(-\frac{\mu}{2}\right) \Phi_{a} \Phi_{a}+\sum_{k=2}^{\infty} \frac{\lambda_{k}}{k}\left(\Phi_{a} * \Phi_{a}\right) * k .
$$

Consideration of other orderings brings no essential modifications since, as we will show, the vanishing of the two point function of the pion field at zero momentum is completely enforced by the chirality of the pion superfield.

For a constant chiral superfield, satisfying the condition $\Phi_{a} \Phi_{a}=a^{2}$, the minimum of the superpotential is achieved for

$$
-\frac{\mu}{2}+\sum_{k=2}^{\infty} \lambda_{k}\left(a^{2}\right)^{k-1}=0
$$

As usual, to break the $O(N)$ symmetry we perform the shift $\Phi_{a} \rightarrow\left(\pi_{i}, \sigma+a\right)$, after which the superpotential can be rewritten as

$$
\begin{aligned}
W\left(\pi_{i}, \sigma\right)= & -\frac{m}{2} \sigma^{2}+\sum_{k=2}^{\infty} \frac{\lambda_{k}}{k}\left(\left[\frac{(2 k) !}{3 !(2 k-3) !} a^{2 k-3} \sigma * \sigma * \sigma\right.\right. \\
& \left.+\frac{(2 k) !}{4 !(2 k-4) !} a^{2 k-4} \sigma * \sigma * \sigma * \sigma\right] \\
& +k a^{2 k-4}\left[2(k-1) a \sigma+\frac{(2 k-2)(2 k-3)}{2} \sigma * \sigma\right] \\
& \left.* \pi_{i} * \pi_{i}+\frac{k(k-1)}{2} a^{2(k-2)}\left(\pi_{i} * \pi_{i}\right)^{2}\right)+\ldots,
\end{aligned}
$$

where only quadratic, cubic, and quartic terms have been explicitly displayed. Then the field $\sigma$ acquires a mass

$$
m=-\mu+\sum_{k=2}^{\infty} \frac{\lambda_{k}}{k} \frac{(2 k) !}{(2 k-2) !} a^{2 k-2},
$$

while the kinetic term takes the form $\int d^{8} z\left(\bar{\pi}_{i} \pi_{i}+\bar{\sigma} \sigma\right)$.

To cancel the divergences we introduce a counterterm action of the form 


$$
\begin{aligned}
S_{c t}= & \int d^{8} z \delta_{z}\left(\bar{\pi}_{i} \pi_{i}+\bar{\sigma} \sigma\right)+\left\{\int d ^ { 6 } z \left[\left(-\frac{\delta_{\mu}}{2}+\sum_{k=2}^{\infty} \frac{\delta \lambda_{k}}{k} \frac{(2 k) !}{2(2 k-2) !} a^{2 k-2}\right) \sigma^{2}+\left(-\delta_{\mu} a+2 \sum_{k=2}^{\infty} \delta \lambda_{k} a^{2 k-1}\right) \sigma\right.\right. \\
& +\left(-\frac{1}{2} \delta_{\mu} a+\sum_{k=2}^{\infty} \delta \lambda_{k} a^{2 k-1}\right) \pi_{i} \pi_{i}+\sum_{k=2}^{\infty} \frac{\delta \lambda_{k}}{k}\left(\left[\frac{(2 k) !}{3 !(2 k-3) !} a^{2 k-3} \sigma^{*} \sigma^{*} \sigma+\frac{(2 k) !}{4 !(2 k-4) !} a^{2 k-4} \sigma * \sigma^{*} \sigma * \sigma+\ldots\right]\right. \\
& \left.\left.\left.+k\left[2(k-1) a^{2 k-3} \sigma+\frac{(2 k-2)(2 k-3)}{2} a^{2 k-4} \sigma^{*} \sigma\right] * \pi_{i} * \pi_{i}+\frac{k(k-1)}{2} a^{2(k-2)}\left(\pi_{i} * \pi_{i}\right)^{2}\right)+\ldots\right]+ \text { H.c. }\right\}
\end{aligned}
$$

The nonvanishing propagators for the chiral superfields $\pi_{i}$ and $\sigma$ read, as usual,

$$
\begin{gathered}
\left\langle\pi_{i} \bar{\pi}_{j}\right\rangle=\delta_{i j} \frac{1}{\square} \delta^{8}\left(z_{1}-z_{2}\right), \\
\langle\sigma \bar{\sigma}\rangle=\frac{1}{\square-m^{2}} \delta^{8}\left(z_{1}-z_{2}\right), \\
\langle\sigma \sigma\rangle=\frac{m D^{2}}{4 \square\left(\square-m^{2}\right)} \delta^{8}\left(z_{1}-z_{2}\right) .
\end{gathered}
$$

There are also $D^{2}, \bar{D}^{2}$ factors associated with the chiral vertices according to the standard Feynman rules in superspace [16]. Observe also that the propagator $\left\langle\pi_{i} \pi_{j}\right\rangle=0$.

The $\left\langle\pi_{i} \bar{\pi}_{j}\right\rangle$ propagator will be represented by a dashed line and the $\langle\sigma \sigma\rangle$ propagator by a solid one. The $(\bigcirc)$ symbol corresponds to a factor $D^{2}\left(\bar{D}^{2}\right)$ associated with the $\langle\bar{\sigma} \bar{\sigma}\rangle(\langle\sigma \sigma\rangle)$ propagator, while all other $D$ factors are associated with vertices by the usual rules in superspace [16]. The trigonometric factors in the vertices have the same structure as in the models seen before. The vertex $\sigma * \pi_{i} * \pi_{j}$ carries a factor

$$
\delta_{i j} \cos \left(p_{1} \wedge p_{2}\right),
$$

while for the vertex $\sigma * \sigma * \sigma$ one finds

$$
\left[\cos \left(p_{1} \wedge p_{2}\right)+\cos \left(p_{2} \wedge p_{3}\right)+\cos \left(p_{1} \wedge p_{3}\right)\right]
$$

where $p_{1}, p_{2}, p_{3}$ are three incoming momenta. The vertex $\pi_{i} * \pi_{j} * \pi_{k} * \pi_{l}$ has a factor

$$
\begin{aligned}
-\lambda & \left\{\delta_{i j} \delta_{k l}\left[\cos \left(p_{1} \wedge p_{2}\right) \cos \left(p_{3} \wedge p_{4}\right)\right]\right. \\
& +\delta_{i k} \delta_{j l}\left[\cos \left(p_{1} \wedge p_{3}\right) \cos \left(p_{2} \wedge p_{4}\right)\right] \\
& \left.+\delta_{i l} \delta_{j k}\left[\cos \left(p_{1} \wedge p_{4}\right) \cos \left(p_{2} \wedge p_{3}\right)\right]\right\},
\end{aligned}
$$

whereas for the vertex $\pi_{i} * \pi_{j} * \sigma * \sigma$ it turns out to be

$$
\delta_{i j} \cos \left(p_{1} \wedge p_{2}\right) \cos \left(p_{3} \wedge p_{4}\right) .
$$

Furthermore, the $\pi$ field mass counterterm and the $\sigma$ tadpole counterterm are, respectively,

$$
-\delta_{i j}\left(-\delta_{\mu} a+2 \sum_{k=2}^{\infty} \delta \lambda_{k} a^{2 k-1}\right)
$$

and

$$
\delta_{\mu} a-2 \sum_{k=2}^{\infty} \delta \lambda_{k} a^{2 k-1}
$$

The gap equation signifying that $\sigma$ has vanishing expectation value is depicted in Fig. 4. Notice that the supergraph including one $\sigma \pi_{i} \pi_{j}$ vertex vanishes because $\left\langle\pi_{i}\left(z_{1}\right) \pi_{j}\left(z_{2}\right)\right\rangle$ $=0$. The gap equation is then the same as in the commutative case. The tadpole graph gives a contribution proportional to

$$
\begin{aligned}
& \left.m \int d^{6} z \sigma(z) \frac{\bar{D}^{2} D^{2} \bar{D}^{2}}{64 \square\left(\square-m^{2}\right)} \delta^{8}\left(z-z^{\prime}\right)\right|_{z=z^{\prime}} \\
& =-\left.m \int d^{8} z \sigma(z) \frac{1}{\left(\square-m^{2}\right)} \delta^{4}\left(x-x^{\prime}\right)\right|_{x=x^{\prime}} \\
& \quad=0,
\end{aligned}
$$

which vanishes as in the commutative case. The vanishing is due only to supersymmetry, since all noncommutative trigometric factors have disappeared. Therefore, vanishing of the counterterm contribution leads to

$$
\delta_{\mu} a-2 \sum_{k=2}^{\infty} \delta \lambda_{k} a^{2 k-1}=0
$$

fixing the relation among the counterterms. It also coincides with the pion mass counterterm implying that it vanishes.

The only one loop contribution to the pion mass renormalization is given by the supergraph shown in Fig. 5. The contribution of this supergraph turns out to be proportional to

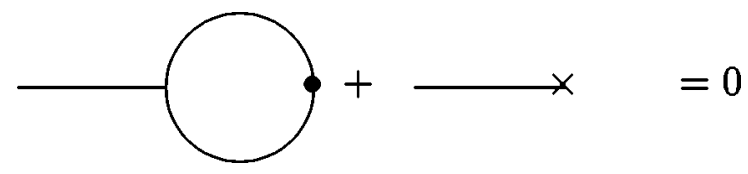

FIG. 4. Gap equation in generic chiral model in $3+1$ dimensions. 


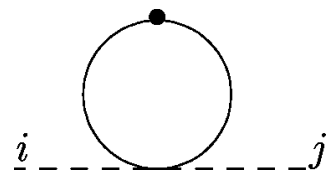

FIG. 5. One loop contribution to the pion two-point function in generic chiral model in $3+1$ dimensions.

$$
\left.\int d^{8} z \pi_{i}(z) \pi_{i}(z) \frac{1}{\left(\square-m^{2}\right)} \delta^{8}\left(z-z^{\prime}\right)\right|_{z=z^{\prime}},
$$

which also vanishes due to the chirality of $\pi_{i}$. Had we used other orderings this expression would contain nontrivial phase factors but, nevertheless, would still vanish for the same reason (i.e., the chirality of the pion field). Therefore we conclude that at one loop order there is no mass renormalization, and this is entirely due to the chirality of the superfield $\pi_{i}$. This is in contrast to the supersymmetric case in three dimensions, where a nonvanishing contribution was found. There the superfields are real and no chirality argument is available.

Just using supersymmetry and chirality arguments allows us to go beyond one loop straightforwardly. At two loops we have to consider the supergraphs with two $\pi_{i}$ external lines which are drawn in Fig. 6. The supergraphs in Figs. 6a, 6b, $6 \mathrm{c}$, and $6 \mathrm{~d}$ contain tadpolelike loop contributions consisting of one propagator $\langle\sigma(z) \sigma(z)\rangle$ or $\langle\bar{\sigma}(z) \bar{\sigma}(z)\rangle$. Since they are proportional to $\left.D^{2} \bar{D}^{2} D^{2} \delta^{8}\left(z-z^{\prime}\right)\right|_{z=z^{\prime}}$ or $\bar{D}^{2} D^{2} \bar{D}^{2} \delta^{8}(z$ $\left.-z^{\prime}\right)\left.\right|_{z=z^{\prime}}$, they both vanish.

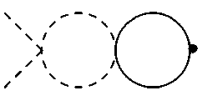

a
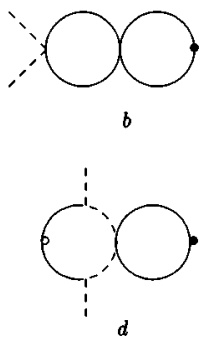

'说'
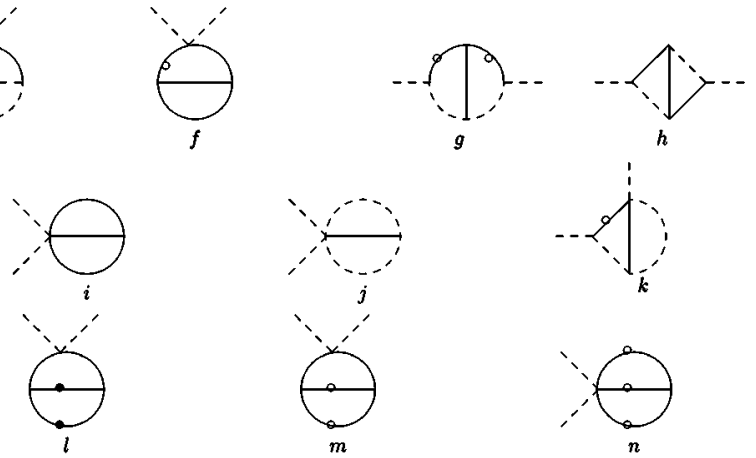

$m$
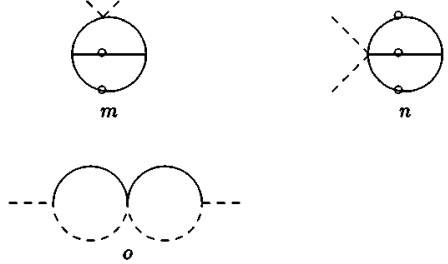

FIG. 6. Two-loop contributions to the pion two-point function in generic chiral model in $3+1$ dimensions.
The supergraphs in Figs. 6e, 6f, 6g, 6h, 6i, 6j, and 6k have equal numbers of $D^{2}$ and $\bar{D}^{2}$ factors; hence, after $D$-algebra transformations, their contributions are proportional to $\int d^{8} z \pi_{i} \pi_{i}$, which is equal to zero due to the chirality of $\pi_{i}$. The supergraphs depicted in Figs. 61, 6m, and 6n are proportional to $D^{2} \delta_{12} D^{2} \delta_{12}$ and to $\bar{D}^{2} \delta_{12} \bar{D}^{2} \delta_{12}$. Both structures give vanishing contributions after integration by parts. Finally, the supergraph drawn in Fig. 6o is proportional to $\int d^{4} \theta \pi_{i} D^{2} \pi_{i}=\int d^{2} \theta \pi_{i} \square \pi_{i}$ and vanishes for slowly varying superfields. We thus conclude that there are no loop corrections to the pion mass for slowing varying superfields. Then the Goldstone theorem is satisfied at one and two loop order for the $O(N)$ supersymmetric chiral superfield whose interactions are compatible with gauge invariance. Notice that the vanishing of these corrections is due to the supersymmetry and not to relations among the coupling constants. In fact, this is a generalization of the nonrenormalization theorem of supersymmetric theories [16] to the noncommutative context.

We can also consider the purely noncommutative interaction (23) in superspace. Its contribution to Eq. (42) is similar to that of (24) but its loop constributions always vanish since $\left\langle\pi_{i} \pi_{j}\right\rangle=0$. So it also is not relevant for the results just derived.

\section{CONCLUSIONS}

In this work, the mechanism of spontaneous symmetry breaking and the appearance of Goldstone bosons were investigated in connection with several noncommutative field models.

We first studied the spontaneous breakdown of the continuous $O(N)$ symmetry in a noncommutative scalar model with quartic and sextuple interactions. For $2+1$ dimensions, there is a class of field orderings for which the model turned out to be renormalizable, up to one loop. The linear combination of couterterms fixed by the gap equation equals the $\pi$-field mass counterterm. Moreover, for the pion two point function, a single condition suffices to ensure (i) cancellation of the UV divergences, (ii) cancellation of the IR divergences arising from the UV-IR mixing, and (iii) the appearance of massless excitations. As shown, this is a peculiarity of the $2+1$ dimensional world. In $3+1$ dimensions, renormalizability called for the elimination of the sextuple interactions while the UV convergence of the planar part restricts the dynamics as already found in [7]. In 2+1 dimensions supersymmetry restricts even further the class of allowed models while, on the other hand, it renders the theory renormalizable to all orders in the limit $N \rightarrow \infty$.

Second, we studied a generic chiral model in $3+1$ dimensions with a local $O(N)$ gauge theory. We argue that this model, although not renormalizable by power counting, may provide a realistic description of the low energy limit of compactified string or $\mathrm{M}$ theory. There are no one loop corrections to the pion mass, while the same holds for two loops in the limit of slowly varying superfields. Supersymmetry enabled us to go through the $N=2$ barrier existing for purely bosonic interactions [7]. 
We also found a purely noncommutative interaction which preserves the $O(N)$ symmetry and is present in any dimension. It starts contributing at $N-2$ loops so that it is not relevant for our one loop analysis. It clearly deserves further study to understand its properties.

Also relevant is the phenomenon of spontaneous breaking of gauge symmetry in noncommutative supersymmetric gauge theories. Its study is in progress.

\section{ACKNOWLEDGMENTS}

This work was partially supported by Fundação de Amparo à Pesquisa do Estado de São Paulo (FAPESP) and Conselho Nacional de Desenvolvimento Científico e Tecnológico (CNPq). H.O.G. and V.O.R. also acknowledge support from PRONEX under Contract No. CNPq 66.2002/1998-99. A.Yu.P. was supported by FAPESP, Project No. 00/12671-7.
[1] R. Szabo, "Quantum field theory on noncommutative spaces," hep-th/0109162; M. Douglas and N.A. Nekrasov, Rev. Mod. Phys. 73, 977 (2001); V. O. Rivelles, Braz. J. Phys. 31, 255 (2001).

[2] S. Minwalla, M. van Raamsdonk, and N. Seiberg, J. High Energy Phys. 02, 020 (2000).

[3] H.O. Girotti, M. Gomes, V.O. Rivelles, and A.J. da Silva, Nucl. Phys. B587, 299 (2000); J. High Energy Phys. 05, 040 (2002); I.L. Buchbinder, M. Gomes, A. Y. Petrov, and V.O. Rivelles, Phys. Lett. B 517, 191 (2001).

[4] H.O. Girotti, M. Gomes, A.Y. Petrov, V.O. Rivelles, and A.J. da Silva, Int. J. Mod. Phys. A 17, 1503 (2002); Phys. Lett. B 521, 119 (2001).

[5] A. Matusis, L. Susskind, and N. Toumbas, J. High Energy Phys. 12, 002 (2000).

[6] F. Ruiz Ruiz, Phys. Lett. B 502, 274 (2001).

[7] B.A. Campbell and K. Kaminsky, Nucl. Phys. B581, 240 (2000); B606, 613 (2001).
[8] F.J. Petriello, Nucl. Phys. B601, 169 (2001).

[9] F. Ruiz Ruiz, Nucl. Phys. B637, 143 (2002).

[10] Y. Liao, Nucl. Phys. B635, 505 (2002).

[11] S. Sarkar and B. Sathiapalan, J. High Energy Phys. 05, 049 (2001); S. Sarkar, ibid. 06, 003 (2002).

[12] E. Katehou and G.G. Ross, Nucl. Phys. B299, 484 (1988).

[13] M. Cvetic, L.L. Everett, and J. Wang, Nucl. Phys. B538, 52 (1999).

[14] A.A. Tseytlin, "Born-Infeld Action, Supersymmetry and String Theory," hep-th/9908105.

[15] S.M. Kuzenko and I.N. McArthur, Phys. Lett. B 522, 320 (2001).

[16] I.L. Buchbinder and S.M. Kuzenko, Ideas and Methods of Supersymmetry and Supergravity (IOP Publishing, Bristol, 1998).

[17] I.L. Buchbinder and A.Y. Petrov, Yad. Fiz. 63, 1745 (2000) [Phys. At. Nucl. 63, 1657 (2000)]; Phys. Lett. B 461, 209 (1999); I.L. Buchbinder, M. Cvetic, and A.Y. Petrov, Nucl. Phys. B571, 358 (2000). 